\title{
Efectos económicos de los movimientos - migratorios en la sociedad de origen y en la de destino
}

Alberto García Sánchez*

\section{Introducción}

\section{$\mathrm{E}$} xiste una gran controversia en cuanto a los efectos beneficiosos o maliciosos de las migraciones tanto en las comunidades de origen como en los lugares de destino. Muchas veces, los argumentos generados en estos debates son utilizados por distintos grupos de poder para apoyar o facilitar la migración a aquellas personas que lo deseen, así como su inclusión social; o bien endurecer las leyes de extranjería y la construcción de fronteras físicas para reducir los flujos migratorios. Este es un debate que, si bien está fundamentado en distintos argumentos que pueden provenir de la misma evidencia empírica, se encuentra fuertemente cargado de ideología. Por tanto, en la mayoría de las veces que se genera, estaría entrando en juego no solo la opinión objetiva ante la migración, sino la propia postura subjetiva ante el funcionamiento de la sociedad y los cambios que puedan experimentarse en ella. No obstante, y como vamos a ver a continuación, la migración llevaría asociada efectos económicos beneficiosos y maliciosos, tanto en el lugar de origen como en el destino; si bien podemos afirmar que, en suma, el migrar parece ser positivo para un lugar y otro. En concreto, para los principales afectados, que son los propios migrantes y sus familiares -migren o no con él-, los potenciales efectos beneficiosos se harían notar en el volumen ingresos, el consumo, la educación o la salud. 
Hay que considerar que los distintos efectos son complejos y susceptibles de cambios con el transcurso del tiempo. Además, el debate de la migración y sus impactos puede englobarse en otro más grande acerca del desarrollo, y más concretamente del codesarrollo; ${ }^{1}$ el cual ha demostrado que no existe un único camino que pueda llevar a un país o región a desarrollarse, con lo que resulta extremadamente difícil sacar conclusiones válidas universalmente con respecto a este tema. También hay que añadir que los efectos derivados de la migración, tanto en origen como en destino, se pueden apreciar más en ámbitos locales que en el total de la nación, debido principalmente a que las zonas que se suelen ver implicadas están claramente delimitadas y pocas veces abarcan al conjunto de un país.

Sobre los efectos económicos de las migraciones se ha investigado ampliamente tanto en los lugares de origen como en los de destino, si bien es en estos últimos en dónde existe una mayor cantidad de estudios. Esto se debe principalmente al interés -preocupación muchas veces- de las sociedades avanzadas en evaluar las consecuencias de incorporar a una nueva población de trabajadores extranjeros en su economía.

Por lo general, el querer mejorar la economía personal o familiar es uno de los motivos por los que las personas deciden migrar y algunos economistas como Dilip Ratha (2010) estiman que esta sería la motivación primordial para nueve de cada diez migrantes internacionales. Es, por tanto, lógico pensar que uno de los principales efectos que conlleva el fenómeno migratorio es el de la transformación de la economía personal, familiar y de las comunidades de origen; pero también hay que tener presente que, en cierta medida, también se transforma la economía en el destino. $Y$ es que se suele asumir que los migrantes se trasladan a sitios donde son más productivos, por lo que a la vez que mejorarían su propia economía también lo haría la de aquellos que les contratan.

1. Según Sami Naïr, creador del concepto, una política de 'codesarrollo' sería aquella que trataría de reforzar la integración de los migrantes en los países de destino a la vez que buscaría favorecer una solidaridad activa con los países de origen, creando las condiciones sociales que permitan a los potenciales migrantes quedarse en sus países si así lo deseen. Sami Naïr, Rapport de bilan et d'orientation sur la politique de codévelopment liée aus flux migratoires, (París: Ministère des Affaires Etrangères, 1997). 


\section{En la sociedad de origen}

Cuando hablamos de efectos económicos de las migraciones para la sociedad de origen estaríamos haciendo referencia, fundamentalmente, a todo aquello derivado del envío de remesas económicas. Aunque existen también otros efectos no menos importantes, como la transformación del mercado laboral debido a la ausencia de personas en edad de trabajar, además de otras consecuencias que también vamos a tratar en este apartado.

Según estimaciones divulgadas por el Banco Mundial, ${ }^{2}$ en 2013 se habrían enviado US $\$ 550.000$ millones en forma de remesas formales en todo el mundo, de ellos US \$ 414.000 millones únicamente hacia países en desarrollo. También predice que se alcanzará para el año 2016 la cifra record de US \$ 707.000 millones en envíos. Todo ello sin contar el volumen de las remesas informales, las cuales son especialmente importantes en los giros realizados entre países del sur, así como en el interior de estos mismos países. Según la clasificación del Banco Mundial, los países que más remesas habrían recibido en 2013 fueron la India con US \$ 71.000 millones, seguido de China con US \$ 60.000 millones, Filipinas US \$ 26.000 millones, México US \$ 22.000 millones, Nigeria US \$ 21.000 millones y Egipto US \$ 20.000 millones. Además, las remesas recibidas habrían constituido el 48\% del PIB en Tayikistán, el 31\% en la República Kirguisa, el 25\% de Lesotho y Nepal, y el 24\% en Moldova.

Estas cifras por sí solas pueden dar una idea del enorme impacto a nivel macroeconómico que tienen las remesas en algunos países receptores, en muchos de los cuales son determinantes a la hora de cuadrar la balanza de pagos. También suponen montos de dinero mucho más elevados que los recibidos en concepto de ayudas al desarrollo, concretamente tres veces más según el Banco Mundial, aunque esta proporción resulta muy variable dependiendo del país del que se trate.

Sin embargo, y pese a la enorme magnitud que reflejan las cifras, en donde verdaderamente se pueden apreciar los efectos beneficiosos de las remesas es en la economía de las familias. La evidencia empírica de

2. "Países en desarrollo recibirán más de US \$ 410.000 millones en remesas en 2013", Banco Mundial (Washington), 2 de octubre de 2013. 〈http://www.bancomundial.org/es/news/press-release/2013/10/02/ developing-countries-remittances-2013-world-bank〉. 
la mayoría de las investigaciones refleja que las remesas sirven para disminuir la pobreza, así lo afirma por ejemplo un estudio realizado por Adams Jr. y Page, ${ }^{3}$ en el que se analiza la relación entre migración internacional, recibimiento de remesas y la disminución de pobreza en 71 países en desarrollo. En él se demuestra que, en promedio, un aumento del $10 \%$ en la proporción de migrantes internacionales de un país, llevaría aparejado una disminución de un 2,1\% en la proporción de personas que vivirían con menos de un dólar al día y hasta de un 3,5\% de aquellas que vivirían en la pobreza gracias, principalmente, al envío de remesas.

Si bien existe unanimidad a este respecto, no parece haber sido tan clara la relación entre migración y mejoramiento de la economía del país receptor, ya que existen muchos autores que destacan el posible estancamiento de la economía debido, precisamente, al recibimiento de esos giros. En este sentido, el economista del Banco de España, Luis Molina, ${ }^{4}$ destaca que uno de los efectos más característicos de la fuerte entrada de divisas sería el aumento del tipo de cambio y, con ello, de los precios relativos de los distintos sectores productivos de la economía del país -se trataría de un proceso similar a lo que se conoce en economía como 'enfermedad holandesa' ${ }^{5}$. El autor afirma que la entrada de remesas daría lugar a un incremento de la demanda por parte de las familias receptoras tanto de bienes comerciables -productos manufacturados-, como no comerciables -comercio minorista, servicios, construcción y productos alimenticios básicos-, que provocaría finalmente una "presión al alza sobre los precios relativos del sector no comerciable frente al comerciable, que está sometido a la competencia exterior" ${ }^{\prime}{ }^{6}$

3. Richard Adams Jr. y John Page, "Do international migration and remittances reduce poverty in developing countries?" World Development 33, No. 10 (2005): 1645-69.

4. Luis Molina, "El efecto de las remesas sobre el tipo de cambio real", Boletín Económico (Banco de España, 2007), 102-11.

5. Este efecto, la 'enfermedad holandesa', 'síndrome holandés' o 'mal holandés', se refiere a las consecuencias potencialmente dañinas para la economía de un país derivadas de una repentina gran entrada de divisas. El término fue utilizado originalmente para describir los efectos que tuvo el descubrimiento de grandes yacimientos de gas natural en los Países Bajos en la década de los sesenta, lo cual provocó, como decimos, una fuerte entrada de ingresos que apreciaría el florín perjudicando con ello a todas las demás exportaciones del país y debilitando, por tanto, a su sector productivo Christine Ebrahim-zadeh, "El síndrome holandés: Demasiada riqueza malgastada", Finanzas \& Desarrollo, (2003): 50-51.

6. Luis Molina, "El efecto de las remesas sobre el tipo de cambio real", 2007. 
Dejando de lado el efecto de las remesas en las tasas de cambio de las divisas, tampoco parecen estar claros los efectos económicos más generales. Así tenemos a autores como Jorge Durand y Douglas Massey que han cambiado de opinión con respecto a esta cuestión con el paso del tiempo. Y es que estosen un principio, al estudiar el caso de la migración mexicana en Estados Unidos, consideraban que las remesas tenían efectos meramente paliativos en la economía de los países de origen ${ }^{7}$ sin embargo, en una investigación posterior concluyeron que los 'migra-dólares' enviados a México sí estimularían la actividad económica, tanto directa como indirectamente, y que llevarían a "niveles significativamente más altos de empleo, inversión e ingresos en el interior de las comunidades específicas y la nación en su conjunto". ${ }^{8}$

No obstante, no se puede afirmar de una manera general que las remesas impulsen el desarrollo, sino que, al igual que los flujos de dinero recibidos en concepto de ayuda al desarrollo del extranjero, estas por sí solas no podrían superar las limitaciones estructurales dentro de los países, las cuales limitarían el crecimiento económico, estancando el cambio y el progreso social. ${ }^{9}$ En esta línea Bettin y Zazzaro, ${ }^{10}$ tras medir la eficiencia de los sistemas bancarios nacionales en sesenta y seis países en desarrollo, afirman que las remesas solo promoverían el crecimiento en aquellos países cuyo sector financiero funcione bien.

Además de los económicos estructurales, otro obstáculo que se aprecia para que las remesas contribuyan al desarrollo es el precio de los giros, el cual suele ser mayor en los envíos que se realizan entre países del Sur. No obstante, según datos aportados por el Banco Mundial (2014), esta situación está cambiando y el coste promedio de los envíos se encontraría, en la actualidad, en una tendencia a la baja en todas las regiones del mundo, entre las cuales, la región latinoamericana sería al día

7. Jorge Durand y Douglas S Massey, "Mexican migration to the United States: A critical review". Latin American Research Review 27, No. 2 (1992): 3-42.

8. Jorge Durand, Emilio A Parrado y Douglas S Massey, "Migradollars and development: A reconsideration of the mexican case", International Migration Review 30, No. 2 (1996): 423.

9. Programa de las Naciones Unidas para el Desarrollo [PNUD], Informe sobre Desarrollo Humano. Superando barreras: movilidad y desarrollo humano. (Madrid: Mundi-Prensa, 2009), 245.

10. Giulia Bettin y Alberto Zazzaro, "Remittances and financial development: Substitutes or complements in economic growth?". Bulletin of Economic Research 64, No. 4 (2012): 509-36. 
de hoy la más barata para enviar remesas y el África subsahariana la más cara.

Otro problema que aumenta el coste de recibir las remesas en las zonas adonde se las envía es el de la distancia que tienen que cubrir los destinatarios para poder cobrarlas. No obstante, cada vez existen más formas de envío y cobro de remesas como el llamado sistema M-PESA, un sistema creado por Safaricom y Vodafone puesto en práctica por primera vez en Kenia en 2007. Este servicio, que permite depositar, enviar y retirar fondos utilizando un teléfono móvil realizando transferencias a través de mensajes SMS a cualquier otro usuario, ha supuesto una experiencia tremendamente exitosa ya que, en muy poco tiempo, a finales de 2009, ya había alcanzado al 65\% de los hogares en aquel país. ${ }^{11}$ Después de Kenia el sistema ha entrado también en los mercados de otros países en desarrollo como República Democrática del Congo, Egipto, Fiyi, India, Lesoto, Mozambique, Sudáfrica y Tanzania, y se espera que en los próximos años se adentre en muchos más. ${ }^{12}$

Dejando de lado ahora los aspectos estructurales y macroeconómicos, vamos a volver a los relacionados con la economía familiar y de las comunidades. En este sentido, los estudios empíricos suelen coincidir en que las remesas sirven para mejorar el nivel de vida de millones de personas en todo el mundo, contribuyendo a que las familias puedan disfrutar de una mejor alimentación, salud y bienestar. ${ }^{13}$ Además, como indica Hans Timmer, director del Grupo de Perspectivas del Desarrollo del Banco Mundial, cada vez parece más claro que los migrantes y sus envíos de dinero están contribuyendo decisivamente a que sus países avancen hacia los llamados objetivos de desarrollo del milenio (ODM), como por ejemplo, conseguir el acceso a la educación, agua potable, saneamiento y atención médica. ${ }^{14}$

11. William Jack y Tavneet Suri, "Mobile money: The Economics of M-PESA", Working Paper, No. 16721 (Cambridge: National Bureau of Economic Research, 2011).

12. "Vodafone and MoneyGram Global Agreement", MoneyGram (Dallas), 11 de febrero de 2014. 〈http:/ /ir.moneygram.com/ releasedetail.cfm?releaseid=824644〉.

13. Dean Yang, "International migration and human development", Human Development Research Paper, No. 29, (Nueva York: Programa de las Naciones Unidas para el Desarrollo [PNUD], 2009).

14. "Países en desarrollo recibirán más de US \$ 410.000 millones en remesas en 2013", 2013. 
Además de ser paliativas, estas mejoras pueden tener un beneficioso efecto a largo plazo. Ya que, como demuestra un estudio longitudinal elaborado con datos empíricos recogidos a lo largo de treinta y cinco años en Guatemala, la mejora en las condiciones de vida y alimentación a edades tempranas provocaría un efecto positivo en el desarrollo cognitivo no verbal en el futuro, ${ }^{15}$ incrementando con ello el capital humano y, consiguientemente, las probabilidades de aumentar los ingresos futuros. ${ }^{16}$ También las remesas sirven a las familias para diversificar las fuentes de ingreso, de modo que estas puedan disponer de un colchón económico a la hora de afrontar imprevistos. ${ }^{17}$ Además, pueden emplearse como ayuda directa en situaciones excepcionales y se tiene evidencia de que los giros han ayudado a sobrevivir a comunidades enteras en conflictos recientes como los de Bosnia Herzegovina, Guinea Bissau, Nicaragua, Tayikistán y Uganda. ${ }^{18}$ No obstante, y como es lógico, el flujo de remesas puede disminuir si los migrantes trabajadores afrontan dificultades económicas en los países donde se encuentran. Aun así, los envíos continúan, aunque en menor cantidad que en otros momentos en los que la situación económica y laboral es mejor.

Se calcula que, en condiciones normales, casi dos tercios de todos los migrantes latinoamericanos enviarían remesas a sus familias como promedio casi un $10 \%$ de sus ingresos. ${ }^{19} \mathrm{Si}$ bien, como decimos, es esperable que incrementen el monto de los envíos en los casos excepcionales. Además la cantidad y frecuencia de los envíos admiten una gran variabilidad, y como afirman de Haas y Plug, el envío de remesas vendría determinado "por factores tales como el número de migrantes, su estado civil, su actividad económica y los ingresos en el país anfitrión y

15. En psicología este aumento de la inteligencia y las capacidades cognitivas en las siguientes generaciones, debido a múltiples causas como puede ser la mejora en la alimentación o en el ambiente en el que se educan los niños, se conoce como 'efecto Flynn'.

16. Jere R Behrman y otros, "What determines adult cognitive skills? Influences of pre-school, school, and post-school experiences in Guatemala", Latin American Economic Review 23, No. 4 (2014).

17. Oded Stark, The migration of labor, (Massachusetts: Basil Blackwell, 1991).

18. Programa de las Naciones Unidas para el Desarrollo [PNUD], Crisis prevention and recovery report 2008: Post-conflict economic recovery, enabling local ingenuity. Nueva York: PNUD, 2008.

19. Comisión Económica para América Latina y el Caribe [CEPAL], Panorama social de América Latina 2004. (Santiago de Chile: Naciones Unidas, 2005). 
de origen, así como sus vínculos afectivos y sociales con su país de origen". ${ }^{20}$

Otro de los factores a tener en cuenta es el género del emisor -se suele afirmar que las mujeres envían una mayor proporción de sus ingresos a casa y más regularmente, pese a que su sueldo normalmente es menor- ${ }^{21}$ pero también del receptor. Por ejemplo, en el caso de Ecuador, las investigadoras Gioconda Herrera y Alexandra Martínez ${ }^{22}$ encontraron que las mujeres receptoras de remesas gastaban más que los hombres en alimentación, salud, vestimenta o educación de los niños, mientras que los hombres empleaban una mayor cantidad de dinero que las mujeres en otros asuntos como el pago de deudas, la construcción o compra de casas, la adquisición de terrenos o automóviles; así como también realizaban más gastos en productos agrícolas y ganado.

La utilidad que se le da a las remesas también estaría condicionada por las necesidades familiares. Así, según Acosta, Fajnzylber y López, ${ }^{23}$ es esperable que en los hogares más pobres se intenten satisfacer previamente las necesidades más básicas, como la alimentación, para cambiar posteriormente los hábitos de gasto conforme vayan superando estas carencias. Estos autores aportan, además, datos que muestran que existen notables diferencias entre países latinoamericanos con respecto a la pobreza de los hogares de las personas que migran. De este modo, exponen que en México y Paraguay habría más hogares receptores de remesas en el quintil más pobre de ingresos que en otros países latinoamericanos -un $61 \%$ en el caso de los mexicanos y un $42 \%$ en el de los paraguayos-. Los países que le seguirían en este ranking serían Ecuador, El Salvador y Guatemala, con al menos un 30\% de los hogares receptores en el quintil con menos ingresos; sin embargo, esta situación cam-

20. Hein de Haas y Roald Plug, "Cherishing the goose with the golden eggs: Trends in migrant remittances from Europe to Morocco 1970-2004". International Migration Review 40, No. 3 (2006): 605.

21. Sarah Murison, “Evaluation of DFID development assistance: Gender equality and women's empowerment. Phase II thematic evaluation: Migration and development", Working Paper No. 13, (Londres: British Government’s Department for International Development, 2005).

22. Gioconda Herrera y Alexandra Martínez, "Género y migración en la región sur", Informe de trabajo de campo, (Quito: FLACSO, 2002).

23. Pablo Acosta, Pablo Fajnzylber y J Humberto López, "Remittances and household behavior: Evidence for Latin America", en Pablo Fajnzylber y J Humberto López, edit., Remittances and Development. Lessons from Latin America (Washington D.C.: Banco Mundial, 2008): 133-70. 
biaría radicalmente en otros países como Perú, en donde al parecer el $40 \%$ de los hogares que reciben remesas pertenecen al quintil más rico, y Nicaragua, donde serían el 33\% de los hogares. En otros países como Bolivia, Honduras, la República Dominicana y Haití, los destinatarios se encontrarían más repartidos entre los quintiles, presentando una distribución en forma de U.

Lo anteriormente dicho vendría a confirmar el hecho de que no migran las personas más pobres de un país. Además, sabemos que tampoco migran desde los países más pobres, y en aquellos en donde lo hacen, las remesas tendrían, al parecer, más efecto para aliviar la pobreza transitoria que la estructural. ${ }^{24}$ Esto podría deberse a que el gasto de las remesas -y su capacidad para potenciar el crecimiento económico- tiende a dirigirse hacia las regiones urbanas, teniendo un impacto menor en las comunidades rurales. Esto es debido a que estos lugares, como relatan Fuentes y Moreno ${ }^{25}$ para el caso de México, carecerían de infraestructura financiera y comercial para poder aprovechar ese dinero. Además, las remesas podrían contribuir a fortalecer la asimetría económica y la estratificación social, precisamente, por la desigualdad que se produciría entre familias receptoras y aquellas no receptoras; circunstancia que incluso puede derivar en una acumulación de tierras en manos de unos pocos migrantes con éxito, generalmente pertenecientes a las primeras familias que migraron. ${ }^{26}$

Por si esto fuera poco, las remesas además pueden servir como acicate a los gobiernos para no invertir en servicios públicos básicos y descuidar las necesidades de ciertos sectores de la población. ${ }^{27}$ Se deja estos asuntos en manos de los migrantes y sus familiares quienes, por otra parte, han demostrado que son más emprendedores que las familias sin migrantes. ${ }^{28}$

24. Devesh Kapur, "Remittances: The new development mantra?", G-24 Discussion Paper Series No. 29. (Nueva York y Ginebra: United Nations, 2004).

25. Noé Arón Fuentes y Luis Ramón Moreno, "Eficacia de las políticas gubernamentales y de las remesas del exterior en la reactivación de las economías rurales en México", Ra Ximhai 5, No. 1 (2009): 19-28.

26. Rubenstein, H. "Migration, development and remittances in rural Mexico". International Migration 30, No. 2 (1992): 127-53.

27. Myron Weiner, The global migration crisis: Challenge to states and to human rights, (Nueva York: HarperCollins College Publishers, 1995).

28. Dean Yang, "International migration and human development", 2009. 
También puede realizarse emprendimiento de una manera colectiva, desde asociaciones y clubes creados por migrantes, familiares y amigos, que tienen un carácter transnacional. Estos grupos han llevado a cabo numerosos proyectos en todo el mundo para mejorar las infraestructuras de barrios y comunidades que incluyen la construcción de caminos, puentes, instalación de sistemas de agua potable, alcantarillado, pozos, instalación de líneas eléctricas o construcciones de iglesias, centros comunitarios o canchas deportivas. A este tipo de envíos, Goldring ${ }^{29}$ los denominó 'remesas filantrópicas', aunque los proyectos pueden estar también cofinanciados por las distintas administraciones. De este caso, el ejemplo más conocido en la literatura sobre remesas es el programa 'Tres Por Uno', con el cual, el Gobierno Federal mexicano, así como algunas administraciones locales del país, se comprometió a aportar tres pesos para proyectos de desarrollo local por cada uno que aportase el migrante. ${ }^{30}$ Una institución que lleva a cabo numerosos proyectos de codesarrollo con remesas en distintos países latinoamericanos es el Fondo Multilateral de Inversiones [FOMIN] del Banco Interamericano de Desarrollo [BID], el cual ha efectuado con éxito distintas experiencias con remesas e inversiones productivas en Brasil, Perú, México, Bolivia, Colombia, Ecuador, Uruguay, Costa Rica, República Dominicana, El Salvador, Nicaragua y Guyana. ${ }^{31}$

En los últimos años ha habido un especial interés académico en evaluar de qué manera estaba afectando la crisis financiera internacional a los flujos de remesas. Una buena recopilación de estudios de este tipo se puede consultar en la obra de Sirkeci, Cohen y Ratha. ${ }^{32}$ En ella, estos autores señalan que las remesas internacionales resultaron menos afectadas por la crisis -cayeron un 5,2\% en 2009- que los flujos de capital privado. Entre otras razones, debido a que gran parte de los giros que

29. Luin Goldring, "Family and collective remittances to Mexico: A multi-dimensional typology", Development and Change 35, No. 4 (2004): 799-840.

30. Rodolfo García Zamora, "El programa tres por uno de remesas colectivas en México: Lecciones y desafíos", Migraciones Internacionales 4, No. 1 (2007): 165-72.

31. FOMIN, Inversiones Productivas, 2011. 〈http://www.fomin.org/mif/programaseideas/AccesoaFinanciamiento/Remesas/Proyectosderemesas/InversionesProductivas/tabid/265/language/es-ES/Default.aspx>.

32. Ibrahim Sirkeci, Jeffrey H Cohen y Dilip Ratha, Migration and remittances during the global financial crisis and beyond, (Washington D.C.: Banco Mundial, 2012). 
enviaban los migrantes provenían de los ahorros de los trabajadores que llevan ya un tiempo en el país de acogida -los recién llegados tienen que afrontar numerosas dificultades en el nuevo país antes de estabilizar su situación económica- y porque, contrariamente a lo esperado, la migración de retorno no se produjo ni tan siquiera en los lugares más afectados por la crisis. Y es que los trabajadores migrantes habrían encontrado la manera de sortear la crisis y seguir mandando dinero a sus países gracias a la adopción de distintas estrategias empleadas para reducir gastos. ${ }^{33}$

Los envíos volvieron a crecer en el año 2010 y los flujos aumentaron sólidamente en todo el mundo salvo en América Latina y el Caribe, zonas más dependientes de la economía estadounidense. ${ }^{34}$ En estas regiones, además, se produjo una fuerte inflación durante la recesión mundial que, junto con el fortalecimiento de sus monedas locales con respecto al dólar, habría reducido el poder adquisitivo de los hogares receptores. ${ }^{35}$

\section{En la sociedad de destino}

Si bien los estudios que evalúan los impactos económicos en el origen suelen concluir en los grandes efectos beneficiosos que tendría la migración para las comunidades y países de procedencia, la literatura acerca de los efectos de la migración en el destino no suele ser tan benévola con el inmigrante. Y es que comúnmente se pueden encontrar en aquellos trabajos argumentos que sostienen que la migración traería consecuencias negativas para los habitantes nativos en la sociedad de destino. Se suele decir, por ejemplo, que la incorporación de trabajadores inmigrantes al mercado de trabajo contribuye a elevar la tasa de desempleo en el país de acogida, que provoca la reducción de los salarios para el conjunto de los trabajadores debido al incremento de la competencia o que de-

33. Alicia Lynch, "Estrategias de remesas de los emigrantes frente a la crisis" Working Paper, No. 2, (Remesas.org, 2010), Ibrahim Sirkeci, Jeffrey H Cohen y Dilip Ratha, Migration and remittances during the global financial crisis and beyond, 2012.

34. "Países en desarrollo recibirán más de US \$ 410.000 millones en remesas en 2013", 2013.

35. René Maldonado, Natasha Bajuk y María Luisa Hayem, Las remesas a América Latina y el Caribe durante el 2010: Estabilización después de la crisis, (Washington D.C.: Fondo Multilateral de Inversiones/Banco Interamericano de Desarrollo, 2011). 
bilita la calidad de los servicios de seguridad social debido, precisamente, a la saturación del sistema al aumentar la demanda de los mismos. ${ }^{36}$

Como vamos a ver a continuación, estos argumentos solo tendrían parte de razón -además es muy probable que, en los casos que la tuviera, se pudiera explicar más debido a las deficiencias de los propios sistemas productivos y de seguridad social que por el incremento de la población activa- y, en general, los efectos económicos a corto, medio y largo plazo, suelen ser positivos o en todo caso neutros.

El efecto económico más obvio de entre todos los que se producen como consecuencia de la migración en el destino es el incremento de la producción que puede llevar consigo la llegada de personas en edad de trabajar. Estas se incorporarían al mercado laboral aumentando con ello la población activa y también las cotizaciones a los sistemas de seguridad social de los países de acogida. La pregunta aquí, habitualmente, es si los inmigrantes aportan más de lo que reciben a los sistemas de bienestar; una cuestión cuya respuesta no parece ser nada clara. En primer lugar, porque los efectos beneficiosos de la inmigración para un país pueden darse de manera indirecta -como vamos a ver a continuación$\mathrm{y}$, en segundo, debido a que los sistemas de seguridad y bienestar social varían enormemente en prestaciones de un país a otro.

En general se suele afirmar que, ya sean positivos o negativos, los efectos fiscales netos de la inmigración no son muy importantes y que, comparados con el PIB, estos no supondrían más de un $1 \%$ de impacto positivo o negativo ${ }^{37}$ En esta línea, Lee y Miller ${ }^{38}$ estimaron, para el caso de Estados Unidos, que las consecuencias fiscales de modificar el volumen de los flujos migratorios no serían muy significativas y que, además, si bien es cierto que la primera generación de inmigrantes podría suponerle algunas cargas al Estado, las generaciones siguientes podrían generar grandes beneficios fiscales.

36. Adolfo Albo y Juan Luis Ordaz Díaz, "Los efectos económicos de la migración en el país de destino. Los beneficios de la migración mexicana para Estados Unidos", Documento de Trabajo, No. 11/17, (México D.F.: BBVA Bancomer, 2011).

37. Robert Rowthorn, "The fiscal impact of immigration on the advanced economies". Oxford Review of Economic Policy 24, No. 3 (2008): 560-80.

38. Ronald Lee y Timothy Miller, "Immigration, social security, and broader fiscal impacts". American Economic Review 90, No. 2 (2000): 350-54. 
Las conclusiones son similares en las investigaciones referentes a los efectos de la inmigración en Europa y, por ejemplo, en un estudio dado a conocer por la Comisión Europea ${ }^{39}$ se defendía la libre circulación de los ciudadanos europeos dentro de la Unión debido a los efectos positivos que traería para la economía del continente en su conjunto. Entre ellos: el estudio afirmaba que los inmigrantes presentarían, por lo general, mayores tasas de empleo que los nacionales, un $67,7 \%$ frente a $64,6 \%$; por otra parte, en la mayoría de veces se trata de personas que vienen con experiencia laboral, por lo que estarían ya formadas; además, la presencia de inmigrantes sin empleo -estudiantes, jubilados, desempleados, etc.- supondría solo una pequeña parte del total de la población extranjera y los gastos en promedio asociados a la asistencia sanitaria de personas desempleadas supondrían solo un $0,2 \%$ del gasto sanitario total. Por último, la Comisión Europea afirma también que la libre circulación de trabajadores forma parte del éxito de los tratados comerciales europeos, debido a que estimula el crecimiento económico al facilitar los viajes y compras transfronterizas.

Por lo general, se asume que la migración internacional contribuye a crear una especialización de los individuos en los países de igual manera a como se produce en el comercio internacional y que esto tiene consecuencias macroeconómicas positivas. ${ }^{40}$ Sin embargo, con respecto a la posible disminución de los salarios debido a la competencia generada por los inmigrantes, los estudios empíricos muestran resultados similares a lo que hemos descrito. Y es que, por lo general, los inmigrantes vendrían a ocupar puestos de trabajo que habrían sido dejados de lado por la población nativa. Así, pese a la llegada de miles de trabajadores foráneos dispuestos a cobrar un menor salario por su trabajo, los estudios realizados en países de la OCDE afirman que el efecto de la inmigración en los salarios de los trabajadores nativos, ya sea positivo o negativo, suele ser insignificante, tanto a corto como a largo plazo. ${ }^{41}$

39. Comisión Europea, Free movement of EU citizens and their families: Five actions to make a differen$\mathrm{ce}$, Communication from the commision to the european parliament, the council, the european economic and social committee and the committee of the regions, (Bruselas: Comisión Europea, 2013).

40. Programa de las Naciones Unidas para el Desarrollo [PNUD], Informe sobre Desarrollo Humano, 2009.

41. Raquel Carrasco, Juan Francisco Jimeno Serrano y Ana Carolina Ortega. "Los efectos de la inmigración sobre las condiciones de los trabajadores nativos en el mercado de trabajo: Evidencia 
Otras investigaciones directamente concluyen que las migraciones estimulan a corto plazo el crecimiento económico en el país de acogi$\mathrm{da},{ }^{42}$ y que incluso podría aumentar los niveles de empleo a la vez que la inversión sin desplazar por ello a los nacionales. No obstante hay que decir que, generalmente, esto depende no tanto del número de los que vengan como del grado de especialización que posean. Y es que cuando los inmigrantes complementan a los nacionales en especialización ambos grupos se podrían beneficiar; sin embargo, cuando compiten por los mismos trabajos sí que se podría ver afectado alguno de los dos grupos dependiendo de los intereses que tenga el empleador. Así por ejemplo, la población nativa con menos formación puede ver en los inmigrantes una fuerte competencia para acceder a empleos de baja cualificación en el sector informal mientras que estos últimos pueden sentir cómo el racismo, manifiesto o encubierto, les cierra la puerta para acceder, a muchos de ellos, a los empleos para los que están cualificados.

Otro efecto de la inmigración, ampliamente documentado y al que se suele referir en casi todas las investigaciones que tratan este asunto, es el de que esta ayudaría a la inclusión de la mujer en el mercado laboral. Esto es debido a que la llegada de trabajadores extranjeros les habría ayudado a liberarse, parcial o totalmente, de otras responsabilidades que tradicionalmente han tenido asignadas como pueden ser las del cuidado de personas mayores, de niños o la realización de tareas domésticas. Lo que relatamos sería particularmente importante en el caso de aquellas que cuentan con mayor formación y perspectivas de ascenso laboral, como recogen para el caso de España Farré, González y Ortega. ${ }^{43}$

para España", en Eliseo Aja Fernández, Joaquín Arango Vila-Belda y Josep Oliver i Alonso, edit., La inmigración en la encrucijada, 158-71. (Barcelona: Bellaterra, 2008). Simonetta Longhi, Peter Nijkamp y Jacques Poot, "A meta-analytic assessment of the effect of immigration on wages", Discussion Papers 134, No. 3, (Hamilton: Tinbergen Institute, 2004). Gianmarco Ottaviano y Giovanni Peri, "Immigration and national wages: Clarifying the theory and the empirics", Working Paper, No. 14188, (Massachusetts: National Bureau of Economic Research, 2008).

42. Francesc Ortega y Giovanni Peri, "The causes and effects of international labor mobility: Evidence from OECD countries 1980-2005", Human Development Research Paper, No. 6, (Nueva York: Programa de las Naciones Unidas para el Desarrollo, 2009).

43. Lídia Farré, Libertad González y Francesc Ortega, "Immigration, family responsibilities and the labor supply of skilled native women", Working Paper, AD series, No. 19, (Valencia: Instituto Valenciano de Investigaciones Económicas, 2009). 
Como venimos diciendo, los inmigrantes estarían más dispuestos que los nacionales a aceptar los trabajos que estos últimos no quieren asumir y que, además, en sociedades avanzadas, estarían cada vez más demandados -como el cuidado de personas mayores-. Por otra parte, a esta flexibilidad a la hora de aceptar un empleo hay que añadir que los inmigrantes también suelen presentar mayor flexibilidad espacial, no importándoles tanto el desplazarse a otras regiones, con lo que pueden ayudar a reducir los desequilibrios regionales. ${ }^{44}$

Otra consecuencia económica estudiada que está asociada a la inmigración es la de los posibles efectos de esta sobre el mercado en general de la sociedad de destino. Es lo que se conoce en economía como efecto spillover o 'efecto inducido de demanda' ${ }^{45}$ Este vendría a decir que el mercado de productos se vería alterado, en algunos casos positiva y en otros negativamente, debido a la llegada de nuevos trabajadores que necesitan atender sus necesidades; pero que, precisamente por ello, se podrían ver afectadas terceras personas, por ejemplo, al subir el precio de ciertos productos o bienes.

No obstante, se ha observado, en casi todos los lugares en los que hay inmigración, que además de productos locales, los inmigrantes abren paso a nuevos mercados. En especial a lo que se ha llamado 'bienes de nostalgia' y que serían aquellos provenientes de sus países de origen. Los principales demandantes de estos productos serían los propios inmigrantes, si bien estos pueden irse abriendo camino poco a poco en el mercado local como es el caso de los comercios de comida étnicos. Relacionado con esto último está la formación de los llamados 'enclaves étnicos', ${ }^{46}$ y que se refiere principalmente a la actividad generada en torno a negocios en los que tanto los empleadores, como empleados, así como la mayor parte de sus clientes, serían de la misma nacionalidad o grupo étnico.

44. Catalina Amuedo-Dorantes y Sara de la Rica, "Complements or substitutes? Immigrant and native task specialization in Spain", CReAM Discussion Paper, No. 16/8, (London: Centre for Research and Analysis of Migration, 2008).

45. Rafael de Arce Borda, "El impacto económico de la inmigración en España, 2000-2009: Antes y después de la crisis". ICE: Revista de Economía, No. 854 (2010): 23-35.

46. Roger Waldinger, "The ethnic enclave revisited". International Journal of Urban and Regional Research 17, No. 3 (1993): 444-52. 
Finalmente, y pese a que la mayoría de los envíos de dinero que se hacen a los países de origen corresponden a trabajadores que envían remesas a sus familias, existen algunos usos de los giros de naturaleza ilícita que ha provocado que cada vez se tenga un mayor interés en controlar los llamados 'canales informales'. Estosson, el financiamiento de grupos terroristas, el lavado de dinero y otros delitos de naturaleza financiera que afectan, de manera indirecta, a los países receptores. ${ }^{47}$

\section{Conclusiones}

Los trabajos que hemos revisado demuestran que los movimientos migratorios son muy buenos para la economía de las personas implicadas, y no tan perjudiciales -como se intenta hacer ver desde posturas políticas conservadoras- para las sociedades de acogida. Es más, estos mismos estudios que hemos consultado demuestran lo contrario. Por ejemplo, la llegada de inmigrantes aumenta el número de cotizantes a la seguridad social, por lo que genera ingresos a las arcas del Estado. También estas personas ayudan a rejuvenecer $-\mathrm{o}$ al menos a paliar el problema de envejecimiento- a las sociedades de acogida, ya que llegan con edad de trabajar y suelen tener más hijos que los nativos. Además, en muchos casos, los inmigrantes ocupan sectores de empleo dejados de lado por la población local y la actividad económica que generan ayudaría a crear nuevos puestos de trabajo, tanto para ellos como para la población autóctona.

Por otra parte, en las sociedades de origen los principales efectos económicos son aquellos derivados del envío de remesas; capaces de aliviar la situación de carencia de las familias y permitiéndoles afrontar todo tipo de imprevistos, pero incapaces, por sí mismas, de contribuir al desarrollo de las regiones adonde se envían. Esto parece ser así, si bien hay que admitir que los giros suponen una fuerte entrada de divisas que mueve, aunque de manera limitada, el comercio local, alimentando la actividad económica y generando, por tanto, algunos puestos

47. Dilip Ratha, "Evaluación del impacto de la migración en el desarrollo económico y social, y su relación causa-efecto", Jornadas de la Sociedad Civl Foro Mundial Sobre Migración y Desarrollo 2010. (México DF: Fundación Bancomer, 2010). 
de trabajo y emprendimientos en las zonas donde se reciben. Algo que ocurre principalmente en las ciudades y centros urbanos, puesto que disponen de una mejor infraestructura para aprovechar esta entrada de dinero que las zonas rurales. Sin embargo, las remesas no solamente conllevan efectos positivos; sino que, al parecer, contribuirían a aumentar la brecha económica y social introduciendo una nueva diferencia entre familias con migrantes y otras con ningún miembro en el extranjero. Además, se ha comprobado que la recepción de remesas puede servir de excusa para que los gobiernos no inviertan en el desarrollo de las comunidades de origen de los migrantes, cuando lo que debe de hacer el Estado, así como los distintos gobiernos regionales y autoridades locales, es alentar y apoyar económicamente a los migrantes y sus familiares en las iniciativas de desarrollo que proponen llevar a cabo en sus comunidades de origen.

\section{Bibliografía}

Acosta, Pablo, Pablo Fajnzylber y J. Humberto López. "Remittances and household behavior: Evidence for Latin America". En Remittances and Development, edit. Pablo Fajnzylber y J. Humberto López, 133-170. Washington D.C.: Banco Mundial, 2008.

Adams Jr., Richard H y John Page. "Do international migration and remittances reduce poverty in developing countries?" World Development 33, No. 10 (2005): 1645-69.

Albo, Adolfo y Juan Luis Ordaz Díaz. Los efectos económicos de la migración en el país de destino. Los beneficios de la migración mexicana para Estados Unidos. Documento de Trabajo, No. 11/17, México D.F.: BBVA Bancomer, 2011.

Amuedo-Dorantes, Catalina, y Sara de la Rica. "Complements or substitutes? Immigrant and native task specialization in Spain". CReAM Discussion Paper, No. 16/8, London: Centre for Research and Analysis of Migration, 2008. Behrman, Jere R, y otros. "What determines adult cognitive skills? Influences of pre-school, school, and post-school experiences in Guatemala". Latin American Economic Review 23, No. 4 (2014).

Bettin, Giulia, y Alberto Zazzaro. "Remittances and financial development: Substitutes or complements in economic growth?". Bulletin of Economic Research 64, No. 4 (2012): 509-36.

Carrasco, Raquel, Juan Francisco Jimeno Serrano y Ana Carolina Ortega. "Los efectos de la inmigración sobre las condiciones de los trabajadores nativos en el mercado de trabajo: Evidencia para España". En La inmigración en la encrucijada, edit., Eliseo Aja Fernández, Joaquín Arango Vila-Belda y Josep Oliver i Alonso, 158-171. Barcelona: Bellaterra, 2008.

Comisión Económica para América Latina y el Caribe [CEPAL]. Panorama social de América Latina 2004. Santiago de Chile: Naciones Unidas, 2005. 
Comisión Europea. Free movement of EU citizens and their families: Five actions to make a difference. Communication from the commision to the european parliament, the council, the european economic and social committee and the committee of the regions. Bruselas: Comisión Europea, 2013.

de Arce Borda, Rafael. “El impacto económico de la inmigración en España, 2000-2009: Antes y después de la crisis". ICE: Revista de Economía, No. 854 (2010): 23-35.

de Haas, Hein, y Roald Plug. "Cherising the goose with the golden eggs: Trends in migrant remittances from Europe to Morocco 1970-2004". International Migration Review 40, No. 3 (2006): 603-34.

Durand, Jorge, Emilio A Parrado y Douglas S Massey. "Migradollars and development: A reconsideration of the mexican case". International Migration Review 30, No. 2 (1996): 423-44.

Durand, Jorge y Douglas S Massey. "Mexican migration to the United States: A critical review". Latin American Research Review 27, No. 2 (1992): 3-42.

Ebrahim-zadeh, Christine. "El síndrome holandés: Demasiada riqueza malgastada". Finanzas \& Desarrollo, (2003): 50-51.

Farré, Lídia, Libertad González y Francesc Ortega. "Immigration, family responsibilities and the labor supply of silled native women". Working Paper, AD series, No. 19, Valencia: Instituto Valenciano de Investigaciones Económicas, 2009.

FOMIN. Inversiones Productivas. 2011. 〈http://www.fomin.org/mif/programaseideas/ AccesoaFinanciamiento/Remesas/Proyectosderemesas/InversionesProductivas/tabid/265/language/es-ES/Default.aspx> Fuentes, Noé Arón y Luis Ramón Moreno. "Eficacia de las políticas gubernamentales y de las remesas del exterior en la reactivación de las economías rurales en México". Ra Ximhai 5, No. 1 (2009): 19-28.

García Zamora, Rodolfo. “El programa tres por uno de remesas colectivas en México: Lecciones y desafíos". Migraciones Internacionales 4, No. 1 (2007): 165-72.

Goldring, Luin. "Family and collective remittances to Mexico: A multi-dimensional typology". Development and Change 35, No. 4 (2004): 799-840.

Herrera, Gioconda y Alexandra Martínez. Género y migración en la región sur. Informe de trabajo de campo, Quito: FLACSO, 2002.

Jack, William y Tavneet Suri. "Mobile money: TheEconomics of M-PESA". Working Paper, No. 16721, Cambridge: National Bureau of Economic Research, 2011.

Kapur, Devesh. "Remittances: The new development mantra?". G-24 Discussion Paper Series, No. 29. Nueva York y Ginebra: United Nations, 2004.

Lee, Ronald, y Timothy Miller. "Immigration, social security, and broader fiscal impacts". American Economic Review 90, No. 2 (2000): 350-54.

Longhi, Simonetta, Peter Nijkamp y Jacques Poot. "A meta-analytic assessment of the effect of immigration on wages". Discussion Papers 134, No. 3, Hamilton: Tinbergen Institute, 2004.

Lynch, Alicia. "Estrategias de remesas de los emigrantes frente a la crisis". Working Paper, No. 2, Remesas.org, 2010. 
Maldonado, René, Natasha Bajuk, y María Luisa Hayem. Las remesas a América Latina y el Caribe durante el 2010: Estabilización después de la crisis. Washington D.C.: Fondo Multilatera de Inversiones, Banco Interamericano de Desarrollo, 2011.

Molina, Luis. "El efecto de las remesas sobre el tipo de cambio real". Boletín Económico, 102-11. Banco de España, 2007. Murison, Sarah. "Evaluation of DFID development assistance: Gender equality and women's empowerment: Phase II thematic evaluation: Migration and development". Working Paper, No. 13, Londres: British Government's Department for International Development, 2005.

Naïr, Sami. Rapport de bilan et d'orientation sur la politique de codévelopment liée aux flux migratoires. París: Ministère des Affaires Etrangères, 1997.

Ortega, Francesc y Giovanni Peri. "The causes and effects of international labor mobility: Evidence from OECD countries 1980-2005". Human Development Research Paper No. 6, Nueva York: Programa de las Naciones Unidas para el Desarrollo, 2009.

Ottaviano, Gianmarco y Giovanni Peri. "Immigration and national wages: Clarifying the theory and the empirics", Working Paper, No. 14188, Massachusetts: National Bureau of Economic Research, 2008. Grupo del Banco Mundial. "Países en desarrollo recibirán más de US \$ 410.000 millones en remesas en 2013". Banco Mundial (Washington), 2 de Octubre de 2013. http:/ / www.bancomundial.org/es/news/ press-release/2013/10/02/developing-countries-remittances-2013-world-banks.

Programa de las Naciones Unidas para el Desarrollo [PNUD]. Crisis prevention and recovery report 2008: Post-conflict economic recovery, enabling local ingenuity. Nueva York: PNUD, 2008.

Programa de las Naciones Unidas para el Desarrollo [PNUD]. Informe sobre Desarrollo Humano. Superando barreras: movilidad y desarrollo humanos, 245. Madrid: Mundi-Prensa, s.a., 2009. Ratha, Dilip. "Evaluación del impacto de la migración en el desarrollo económico y social, y su relación causa-efecto". Jornadas de la Sociedad Civl Foro Mundial Sobre Migración y Desarrollo 2010. México D.F.: Fundación Bancomer, 2010.

Rowthorn, Robert. "The fiscal impact of immigration on the advanced economies". Oxford Review of Economic Policy 24, No. 3 (2008): 560-80.

Rubenstein, H. "Migration, development and remittances in rural Mexico". International Migration 30, No. 2 (1992): 127-53.

Sirkeci, Ibrahim, Jeffrey H Cohen y Dilip Ratha. Migration and remittances during the global financial crisis and beyond. Washington D.C.: Banco Mundial, 2012.

Stark, Oded. The migration of labor. Massachusetts: Basil Blackwell, 1991.

MoneyGram. "Vodafone and MoneyGram Global Agreement". MoneyGram (Dallas), 11 de Febrero de 2014. 〈http://ir.moneygram.com/releasedetail.cfm?releaseid $=824644$.

Waldinger, Roger. "The ethnic enclave revisited". International Journal of Urban and Regional Research 17, No. 3 (1993): 444-52.

Weiner, Myron. The global migration crisis: Challenge to states and to human rights. Nueva York: HarperCollins College Publishers, 1995. 
72 - Comentario Internacional • Número dieciséis • año 2016

Yang, Dean. "International migration and human development". Human Development Research Paper, No. 29, Nueva York: Programa de las Naciones Unidas para el Desarrollo [PNUD], 2009. 